\title{
A spatial analysis of the discriminability of forms in noise'
}

\author{
LEONA S. AIKEN AND DONALD R. BROWN 2 PURDUE UNIVERSITY
}

Multidimensional scaling (MDS) techniques were used to study the form characteristics which transcend noise in a discrimination task. Three dimensions of random polygons were identified and were psychophysically related to quantified form measures.

Though the ability of the human observer to separate signal from noise has been extensively investigated in the signal detection paradigm, relatively little research concerning the effects of noise on visual pattern perception has been performed. Most of the research which does exist on this topic, a series of studies which demonstrate that introducing noise into a visual display degrades performance, has been reviewed (Webster, 1966).

The research which has been reported has suffered from several weaknesses, and results have been difficult to compare from study to study. One of the problems has been that noise has been variously defined as deletion or addition of units in the contour of a figure, changes in the state of the matrix grain defining the figure (Fitts, Weinstein, Rappaport, Anderson, \& Leonard, 1956), addition and displacement of cells in the figure (Webster, 1963), decreases in figure-ground contrast, and increases in contour blur (Brown \& LoSasso, 1967).

A second problem has been variation in the matrix grain used both in generating and "noising" figures. Although little investigated, it seems certain that matrix grain size bears an important relation to noise effects, especially when noise involves changes of state in matrix grain; and it is likely that various cells have differential significance to veridical perception of the figure embedded in noise. In the present study, a very fine matrix grain was used both to generate forms and to introduce noise into the display.

Finally, the interesting question of the differential effects of noise upon different form characteristics has received little attention. It has been demonstrated that display characteristics such as complexity (French, 1954), redundancy (Rappaport, 1957a, b), size of noise detail (Baker \& Alluisi, 1962), and type of noise (Munsinger \& Gummerman, 1967; Duke, 1967) differentially affect performance. In an earlier paper (Brown \& Owen, 1967) the exploratory use of multidimensional scaling (MDS) techniques in the study of form perception was discussed; and in subsequent studies, using both similarity estimates (Behrman \& Brown, 1968) and discrimination latencies (Brown \& Andrews, 1968), interpretable MDS results were obtained. The present study was carried out in the same exploratory vein in an attempt to obtain interpretable MDS results using yet another dependent variable and, at the same time, with an interest in examining the nature of form characteristics which transcend noise.

\section{Subjects}

\section{METHOD}

Six male and six female graduate students, paid for their participation, served as Ss.

\section{Stimuli}

Stimuli used herein were 20 eight-sided random polygons (chosen from a larger sample of 200 eight-sided forms), which were generated in a 100 by 100 unit matrix by a modified Method 1 of Attneave and Arnoult (1956). These forms were quantified on 80 physical measures; and the measures were factor analyzed, yielding 12 orthogonal factors of which the first five account for $61 \%$ of the variance in the measures for the eight-sided shapes. This physical measure system has been described in detail by Brown and Owen (1967).

Forms were selected to assure independence among selected physical measures and to assure that the sample of forms covered the range of the measures used for selection. Five measures ${ }^{3}$ were selected on the basis of their high correlation with the first five factors of the factor solution to the measures space (Brown \& Owen, 1967) and their similarity to measures previously found to be psychophysically useful (Behrman \& Brown, 1968; Brown \& Andrews, 1968). For the sample of forms selected, the linear correlations (Pearson, product moment) among the five measures ranged between -.18 and +.34 . The forms and their standardized values on the five measures are shown in Fig. 1.

\section{Problems and Noise}

To create noise in the figure background, a series of 25100 by 100 unit matrices were computer-generated with $2 \%$ noise increments. Beginning with 200 randomly-chosen cells as the first noise matrix, 24 subsequent matrices were generated by sampling 200 cells without replacement in each matrix. The 25 matrices, containing $2-50 \%$ noise, were plotted as black noise cells with a digital incremental plotter and photographed; and the negatives, containing $50-98 \%$ noise, were used to mask the forms. Problems were constructed by photographing each form with the noise overlays. Ten per cent noise was added to the forms by random selection of form cells so that the total noise in the form-on-ground was determined by the intersection of the probability of a noise cell in the form

\begin{tabular}{|c|c|c|c|c|}
\hline \multicolumn{5}{|c|}{ MEASURE } \\
\hline 1 & 2 & 3 & 4 & 5 \\
\hline 3.53 & $\overline{\mathbf{2} .89}$ & $7 \overline{440}$ & $\overline{3.28}$ & $-\overline{2.20}$ \\
\hline 3.39 & $\sim .62$ & L 1.09 & 72.66 & .1 .67 \\
\hline - 1.23 & $\sim .62$ & $\leqslant .64$ & $\begin{array}{l}1.15 \\
\end{array}$ & 71.33 \\
\hline+.83 & $\mathrm{1} .48$ & 28 & $<1.64$ & 11.23 \\
\hline 1.80 & $\leftarrow .43$ & 7.19 & $\begin{array}{l}-1.87 \\
\end{array}$ & .83 \\
\hline$<\quad .55$ & -.21 & $-\quad .10$ & .85 & .35 \\
\hline 49 & -.11 & $y \cdot .17$ & $-\quad .83$ & .28 \\
\hline 、. 41 & $7 \cdot .10$ & A. .24 & 31 & .23 \\
\hline 7.16 & v. -.23 & $>-31$ & .20 & .16 \\
\hline .15 & $1 \cdot .38$ & $<-.39$ & -.14 & .13 \\
\hline 5.11 & -.61 & $4-.42$ & $\star-.04$ & $1-.05$ \\
\hline ㄴ. . .07 & --.65 & $\sim-.66$ & $1 \cdot .13$ & $<-.15$ \\
\hline$\checkmark .05$ & $1-.68$ & 1. .83 & $\checkmark-.22$ & $=-.29$ \\
\hline a -.27 & $<-.71$ & 1. .94 & $\sim-.38$ & - -.47 \\
\hline $7-.36$ & -1.02 & -.95 & $<-.67$ & $=-64$ \\
\hline-.52 & L -1.18 & -1.20 & a -.75 & ( $1 .-23$ \\
\hline-.53 & -1.55 & a -1.58 & L. -.97 & \& -1.28 \\
\hline-.68 & $=-1.60$ & -1.67 & s -1.70 & +-1.31 \\
\hline-.76 & -1.65 & -1.82 & -2.36 & $-\quad-1.91$ \\
\hline$<-1.0$ & -1.1 .70 & - -2.08 & -2.97 & L -2.44 \\
\hline
\end{tabular}

Fig. 1. Ordering of shapes on five selection measures: (1) second moment of combined radials; (2) third moment of interior angles; (3) ratio of vertical to horizontal extent; (4) $X$ coordinate of areal center of gravity; (5) $Y$ coordinate of areal center of gravity. See Brown and Owen (1967) for a more complete description of these measures. 


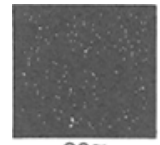

$98 \%$

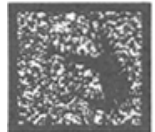

$72 \%$

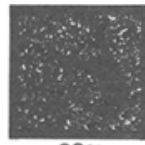

$92 \%$

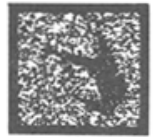

$68 \%$

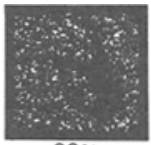

$88 \%$

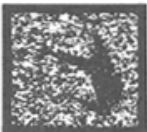

$62 \%$

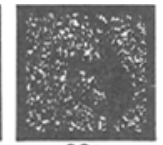

$82 \%$

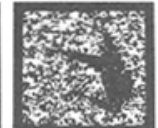

$58 \%$

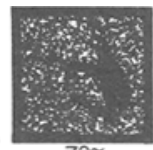

$78 \%$

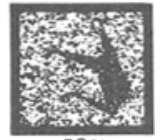

$52 \%$
Fig. 2. Partial noise series for one form.

(white on black) and in the background (black on white) and ranged between $0.2 \%$ to $5.0 \%$ as the noise in the background changed from $98 \%$ to $50 \%$. An example of a form embedded in the noise sequence is shown in Fig. 2.

\section{Apparatus and Procedure}

The experimental equipment consisted of two slide projectors, timing equipment to control stimulus presentation intervals and to measure response latency, and a voice key to deactivate projectors and timers when Ss responded verbally.

Each trial consisted of two phases. A pair of forms was back-projected onto a viewing screen. Each S responded with a similarity judgment ${ }^{4}$ made on a seven-point scale; his response immediately turned off the projected image. The pair was then projected again, and to its left was projected one of the forms embedded in $98 \%$ noise. This slide was followed at 1.5 -sec intervals by slides of progressively decreasing noise which continued until the S responded "right" or "left," indicating which pair member was embedded in noise. Errors occurred infrequently; but when they did occur, that problem was repeated later in the series. The per cent noise at which correct discrimination occurred was recorded as the dependent variable. This procedure was repeated for the 190 possible pairs of forms, which were presented in the order of the Ross pairings (1934). Pairs were balanced with respect to the frequency with which a form appeared on, and was correct on, each slide, under the restriction that no run of more than four correct stimuli could occur on a single side.

Testing was carried out in four 1-h sessions, over a six-day period, in four blocks of 50 trials with 50 practice trials on the first day and the last day devoted to collecting data not reported in this article. To control for practice effects, blocks were administered in a 4 by 4 Latin square design with groups of three Ss each assigned to square entries. With 190 pairs and 200 trials this allowed 10 trials, chosen from across the four days, to be repeated for each $\mathrm{S}$ as a reliability check.

\section{RESULTS}

An analysis of variance of the Latin square control design showed nonsignificant block effects, indicating that blocks of trials were of equal difficulty when averaged over the four days. The day effects were significant $(p \leqslant .01)$, indicating that performance improved over the four-day testing period. Based upon the 10 repeated trials, an average test-retest reliability of $.69(p \leqslant .05)$ was obtained.

A principal-components' analysis of Ss across discrimination trials was performed in order to examine the possibility of meaningful subgroupings of Ss which might be used in the subsequent scaling analysis. All Ss loaded similarly on the first factor, i.e., loadings between .58 and .78; this factor accounted for $48.5 \%$ of the total matrix variance. Loadings on subsequent factors were low and failed to yield clearcut homogeneous groups of Ss.

The major analyses were based upon the assumption that discriminability of forms in noise is inversely and monotonically related to distance in a space, i.e, the more similar two stimuli are, the less noise can be tolerated for accurate discrimination. Accordingly, the mean per cent noise at which forms were discriminated was used as a similarity estimate with the Kruskal nonmetric multidimensional scaling technique (Kruskal, 1964a, b), using Minkowski metrics of $r=1.5$, 2.0, and 3.0. As a first criterion by which to evaluate the scaling solutions, the stress curves were examined. It is clear from Fig. 3 that the various metrics provided about the same degree of fit, that stress was relatively high, and that no clear break in the stress curve occurred to indicate the appropriate dimensionality. Consequently, the criteria of interpretability and psychophysical fit to the physical measures were used to select among the two-, three-, and four-dimensional solutions for each metric. As a first interpretive device, intercorrelations (Pearson, product moment) were computed among the projections of forms on the dimensions of the various solutions and are shown in Table 1 . In Table $1, \mathrm{r}$ denotes the solution metric, and the row and column headings represent the dimensions from the two-, three-, and four-dimensional solutions with the various metrics. The entries are correlations (Pearson, product moment) among projections on the axes and reflect the degree of similarity among solutions. It is clear from these correlations that the solutions of higher dimensionality essentially reproduced solutions of lower dimensionality within each metric and that the similarity of the ordering of forms was high across metrics. For instance, the dimensions of the two-dimensional, $r=1.5$ solution correlated from -.63 to +.96 with dimensions of all other solutions. These correlations, then, indicate a high degree of similarity among the solutions in two through four

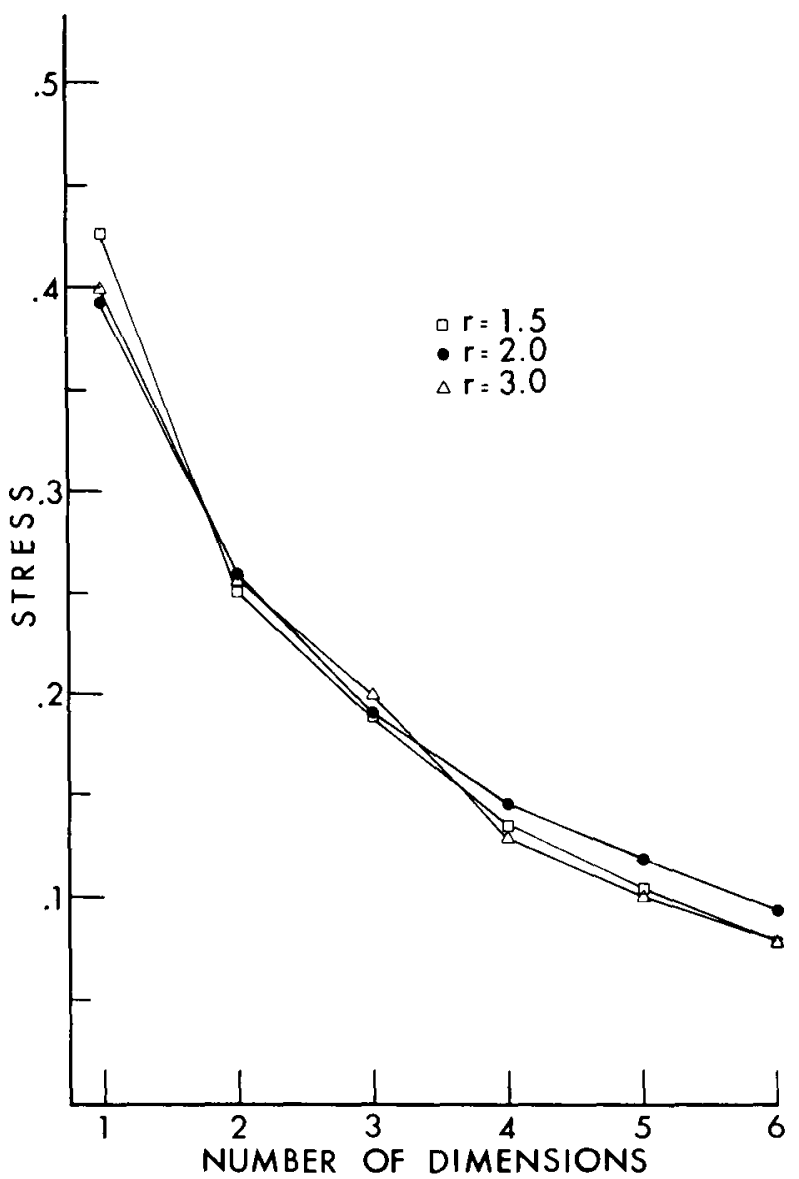

Fig. 3. Values of stress for the three Minkowski solutions, $r=1.5,2.0$, and 3.0 . 
Table 1

Correlation Among Dimensions of Solution Spaces of Varying Dimensionality and Metric

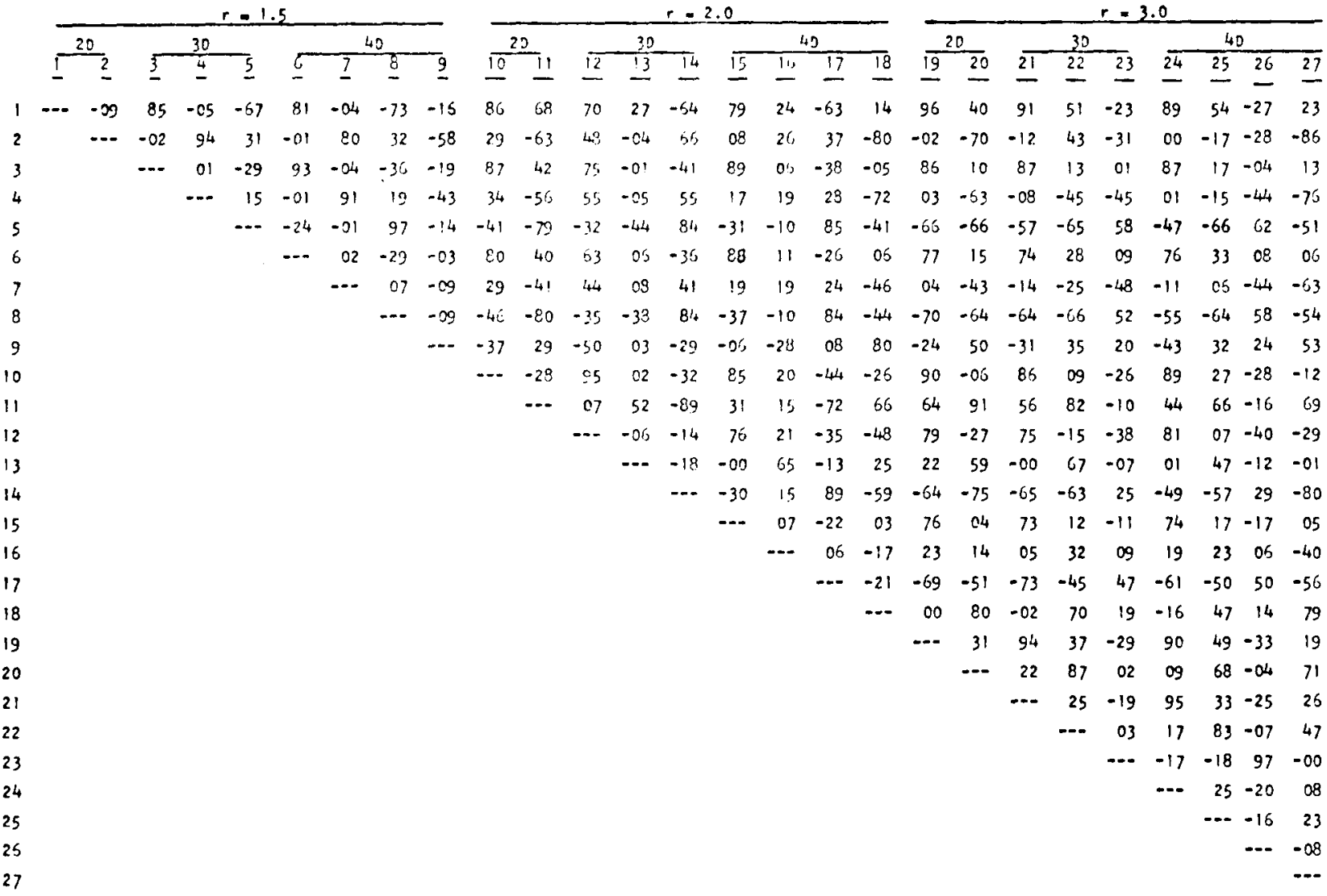

dimensions, and final selection of a solution was referred to a psychophysical analysis.

In an initial psychophysical analysis, five form measures were selected to correlate with the various solutions on the basis of their utility in previous studies (Brown \& Andrews, 1968; Behrman \& Brown, 1968) with four-sided forms. Results were disappointing since the correlations were only moderate and not particularly useful, therefore, for psychophysical prediction. Consequently, the dimensions of all solutions were correlated with all 80 measures available on the forms. On the basis of the similarity among all solutions otherwise, the space selected for final interpretation was the three-dimensional, $r=3$ solution since it gave the highest correlations with plysical measures. The intercorrelations among measures and between measures and dimensions are summarized for this solution in Table 2 .

The three dimensions of the $r=3.0$ solution are shown plotted against the physical measures with which they were most highly correlated in Figs. 4a-c. In these figures, r refers to the correlation between the dimension and the form measure. The first dimension was interpreted as a "jaggedness" dimension since a small interior angle in a form indicates the presence of a sharp protrusion or pinnacle. As seen in Fig. 4a, those forms with low projections on this dimension are jagged in appearance while those with higher projections tend to be smoother. The second dimension was sensitive to the existence of a side length in relatively large proportion to the perimeter length and is shown plotted against this ratio in Fig. 4b. This correlation is relatively low, and it should be noted that two forms which do not fall near the regression line are peculiar in that they contain more than a single long side. This causes the ratio to be relatively small, while the forms do contain obvious long sides. Finally, the third dimension, shown in Fig. $4 \mathrm{c}$ plotted against the variance of the $X$ coordinates, was interpreted as an indicant of horizontal variability. The narrowest form marks the upper end of the distribution, while wide forms mark its lower limit.

\section{DISCUSSION}

The adequacy of a multidimensional scaling solution depends upon a variety of factors including, but probably not limited to, the degree of error in the observations, the number of dimensions, the number of points (Young, 1968), and the choice of a metric. Moreover, the criteria by which adequacy can be assessed include goodness-of-fit measures such as stress, interpretability, and, in special circumstances, the extent to which a nonmetric analysis recovers metric information (Young, 1968). When the $\mathrm{E}$ has an a priori hypothesis or model concerning the dimensionality and metric, the problem of selecting a best solution space is difficult, but relatively simple when compared to the exploratory use of MDS

Table 2

Intercorrelations Among Selected Physical Measures and the ThreeDimensional, $r=3.0$, MDS Results (decimels omitted).

\begin{tabular}{lllrrrr} 
& & \multicolumn{3}{c}{ Measures } & \multicolumn{3}{c}{ Dimensions } \\
& $\mathrm{M}_{1}$ & $\mathrm{M}_{2}$ & $\mathrm{M}_{3}$ & $\mathrm{D}_{1}$ & $\mathrm{D}_{2}$ & $\mathrm{D}_{3}$ \\
$\mathrm{M}_{1}$ & -- & 25 & -03 & 73 & -03 & -22 \\
$\mathrm{M}_{2}$ & & - & 31 & -01 & 49 & -03 \\
$\mathrm{M}_{3}$ & & & -- & 12 & 24 & -66 \\
\hline
\end{tabular}

$M_{1}=$ The size, in degrees, of the smallest interior angle of the form. $M_{2}=$ The ratio of the longest side to the length of the perimeter. $M_{3}=$ The variance of the $X$ coordinates. 


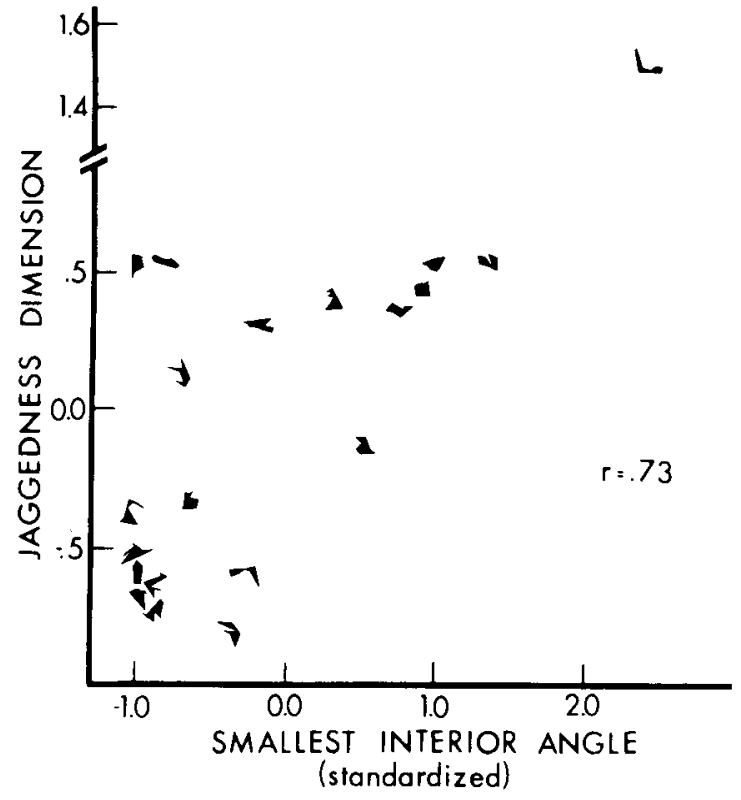

Fig. 4a. Relationship between the jaggedness dimension and the smallest interior angle.

methodology in areas where both the metric and the dimensionality are in question and the degree and distribution of errors are unspecified. Under these latter circumstances, a decision must be based upon a combination of goodness-of-fit and interpretability as criteria.

While it would be desirable to maximize goodness-of-fit (lower stress in the present case), arbitrary criteria of goodness-of-fit can be met simply by increasing the dimensionality of solutions. This approach may be undesirable since uninterpretable dimensions may be obtained (Stenson, 1968), and it seems doubtful that the O can reliably order stimuli with regard to more than a small set of dimensions in any one task (Fenker \& Brown, 1968). In the present study, a relatively high level of stress was tolerated in the final solution. The three-dimensional solution was selected because of its

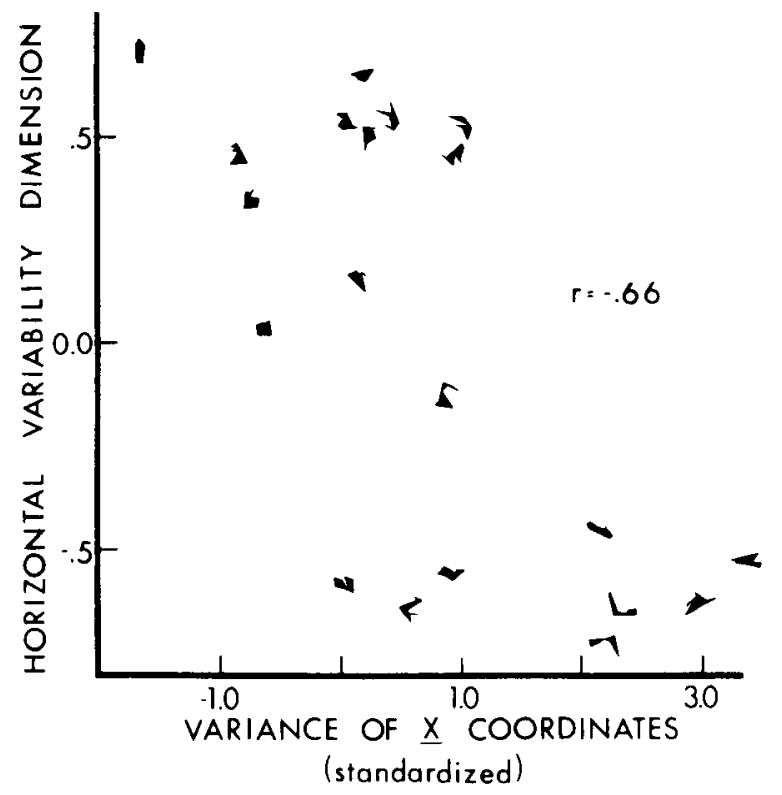

Fig. 4c. Relationship between the horizontal variability dimension and variance of the $X$ coordinates. similarity to previous results with four-sided polygons in a similarity judgment task (Behrman \& Brown, 1968) and a discrimination task (Brown \& Andrews, 1968) and because of its psychophysical interpretability.

The choice of a metric, $r=3.0$, for the solution space presented here was also based upon psychophysical interpretability. To date, the literature on MDS results has largely been limited to the use of either Euclidean $(r=2.0)$ or "city block" $(r=1.0)$ solutions primarily because these metrics provide intuitively appealing and relatively simple spatial models for how differences among stimuli are combined into a total similarity estimate. The "city block" model assumes that Os simply sum differences with regard to all perceptually relevant attributes and has been proposed as a reasoriable model when component dimensions are distinct. This notion has received some support when geometric forms were used as stimuli (Attneave, 1950; Hyman \& Well, 1967, 1968; Torgerson, 1952), as has the idea that a Euclidean solution is more appropriate when component dimensions are less analyzable

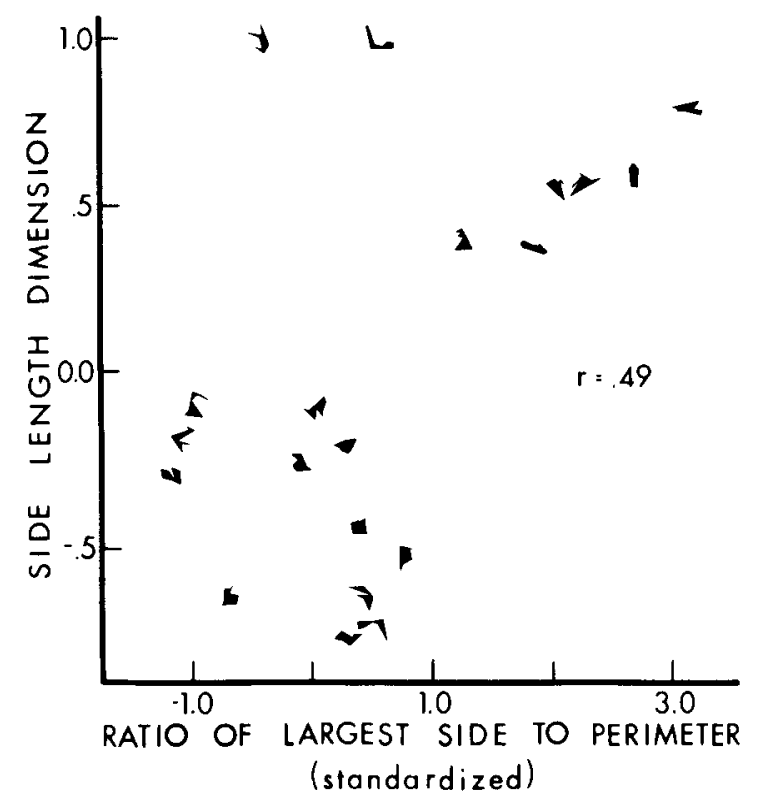

Fig. 4b. Relationship between the side length dimension and the ratio of largest side to perimeter.

(Hyman \& Well, 1967, 1968; Shepard, 1964; Torgerson, 1952) with such stimuli as color. This matter cannot be considered to be resolved. Although our studies have not been designed to examine the metric question in detail, we have consistently found that the "city block" solution yields the poorest fit when random polygons are judged (Behrman \& Brown, 1968) or discriminated (Brown \& Andrews, 1968). Perhaps the dimensions of random polygons are less compelling than those associated with other studies in which the "city block" solution was best. In any event, the higher-order metric, $r=3.0$, was used herein simply because goodness-of-fit was similar for all metrics evaluated, and this solution was most clearly related to the physical measures. This implies that best fit is obtained when more weight is given to larger differences between stimuli. Whether this is a useful model for discrimination behavior remains to be seen.

The general question of using discrimination-in-noise to obtain a spatial solution has been answered affirmatively. While the solution was "noisy," an interpretable solution was obtained; and the form characteristics which transcend noise appear to be similar to those which provide the basis for 
similarity judgments and those which serve as a basis for discrimination in the absence of E-generated noise.

\section{REFERENCES}

ATTNEAVE, F. Dimensions of similarity. American Journal of Psychology, 1950, 63, 516-556.

ATTNEAVE, F., \& ARNOULT, M. D. The quantitative study of shape and pattern recognition. Psychological Bulletin, 1956, 53, 452-471.

BAKER, E. J., \& ALLUISI, E. A. Information handling aspects of visual and auditory form perception. Journal of Engineering Psychology, $1962,1,159-170$

BEHRMAN, B. W., \& BROWN, D. R. Multidimensional scaling of form: A psychophysical analysis. Perception \& Psychophysics, 1968, 4 , 19.25.

BROWN, D. R., \& ANDREWS, M. H. Visual form discrimination: Multidimensional analyses. Perception \& Psychophysics, 1968, 3, 401-406.

BROWN, D. R., \& LoSASSO, J. S. Pattern degradation, discrimination difficulty, and quantified stimulus attributes. Psychonomic Science, $1967,9,351-352$.

BROWN, D. R. \& OWEN, D. H. The metrics of visual form: Methodological dyspepsia. Psychological Bulletin, 1967, 68, 243-259.

DUKE, J. A. Noise methods in pattern perception. Perception \& Psychophysics, 1967, 2, 338-340.

FENKER, R. M., JR, \& BROWN, D. R. A methodology for defining the conceptual space of an individual. Paper presented at the Midwestern Psychological Association, Chicago, May, 1967.

FITTS, P. M., WEINSTEIN, M., RAPPAPORT, M., ANDERSON, N., \& LEONARD, J. A. Stimulus correlates of visual pattern recognition: A probability approach. Journal of Experimental Psychology, 1956, 51, 1-11.

FRENCH, R. S. Pattern recognition in the presence of visual noise. Journal of Experimental Psychology, 1954, 47, 27-31.

HYMAN, R., \& WELL, A. Judgment of similarity and spatial models. Perception \& Psychophysics, 1967, 2, 233-248.

HYMAN, R., \& WELL, A. Perceptual separability and spatial models. Perception \& Psychophysics, 1968, 3, 161-165.

KRUSKAL, J. B. Multidimensional scaling by optimizing goodness of fit to a nonmetric hypothesis. Psychometrika, 1964a, 29, 1-27.

KRUSKAL, J. B. Nonmetric multidimensional scaling: A numerical method. Psychometrika, 1964b, 29, 115-129.

MUNSINGER, H., \& GUMMERMAN, K. Identification of form in patterns of visual noise. Journal of Experimental Psychology, 1967, $75,81-87$.

RAPPAPORT, M. Redundancy as a stimulus parameter in form discrimination. In J. W. Wulfeck and J. H. Taylor (Eds.), Form discrimination as related to military problems. Washington, D. C.: National Academy of Science, No. 561, 1957a, 45-49.

RAPPAPORT, M. The role of redundancy in the discrimination of visual forms. Journal of Experimental Psychology, 1957b, 53, 1-10.
ROSS, R. T. Optimum orders for the presentation of pairs in the method of paired comparisons. Journal of Educational Psychology, 1934, 25 375-382.

SHEPARD, R. N. Attention and the metric structure of stimulus space. Journal of Mathematical Psychology, 1964, 1, 5487.

STENSON, H. H. The psychophysical dimensions of similarity among random shapes. Perception \& Psychophysics, 1968, 3, 201-214.

TORGERSON, W. S. Multidimensional scaling: I. Theory and method Psychometrika, 1952, 17, 401-419.

WEBSTER, R. B. Pattern discrimination as a function of fill, distortion, and noise. Perceptual \& Motor Skills, 1963, 16, 361-367.

WEBSTER, R. B. Stimulus characteristics and effects of fill, distortion, and noise on pattern perception. Perception \& Motor Skills, 1966, 23, 19-33.

YOUNG, F. W. Nonmetric multidimensional scaling: Development of an index of metric determinancy. L. L. Thurstone Psychometric Laboratory Report, Chapel Hill, N.C., No. 56, 1968.

\section{NOTES}

1. This research was supported by Research Grant HD 00909 from the National Institute of Child Health and Human Development.

2. Address: Department of Psychology, Purdue University, Lafayette, Indiana 47907.

3. The measures used to select forms were:

(a) The second moment of radial lengths. A radial was a line connecting the area center of gravity to the perimeter. Radials were computed in 1-deg steps from $0 \mathrm{deg}$ to $360 \mathrm{deg}$, and the second central moment was computed. This essentially measures dispersion of area from a compact figure and is highly correlated with perimeter length in the sample of 200 forms.

(b) Third moment of interior angles. The size of all interior angles was measured in degrees, and their third central moment was computed. This measures "jaggedness," or the degree of skewness of the distribution of angle sizes.

(c) The ratio of vertical to horizontal extent. Vertical extent was computed as the difference between the largest and smallest $Y$ coordinate; horizontal extent was the difference between the largest and smallest $X$ coordinate. The ratio measures degree of elongation with regard to the fixed $X$ and $Y$ reference system.

(d) The $X$ coordinate of the area center of gravity. This measures the location of the mass of area relative to the $X$ axis.

(e) The $Y$ coordinate of area center of gravity. A measure of the location of the mass of area relative to the $\mathrm{Y}$ axis.

4. An analysis of the similarity judgments, as well as discrimination difficulty judgments made on the same pairs after completion of this task, are to be presented in a subsequent paper.

(Accepted for publication October $11,1968$. 